\title{
UNDERSTANDING EMOTION IN RAAG: AN EMPIRICAL STUDY OF LISTENER RESPONSES
}

\author{
Parag Chordia and Alex Rae \\ Georgia Institute of Technology, Department of Music \\ 840 Mc Millan St., Atlanta GA 30332 \\ $\{$ ppc,arae3\}@gatech.edu
}

\begin{abstract}
A survey of emotion in North Indian classical music was undertaken to determine the type and consistency of emotional responses to raag. Participants listened to five oneminute raag excerpts and recorded their emotional responses after each. They were asked to describe the emotions each excerpt evoked and then to adjust six different sliders indicating the degree to which they felt the following: happy, sad, peaceful, tense, romantic, longing. A total of 280 responses were received. We find that both free-response and quantitative judgments of emotions are significantly different for each raag and quite consistent across listeners. We hypothesized that the primary predictors of emotion in these excerpts would be pitch-class distribution, pitch-class dyad entropy, overall sensory dissonance, and note density. Multiple regression analysis was used to determine the most important factors, their relative importance, and their total predictive value $\left(R^{2}\right)$. The features in combination explained between $11 \%$ (peaceful) and $33 \%$ (happy) of response variance. For all models, a subset of the features were significant, with the interplay between "minor" and "major" scale degrees playing an important role. Although the explanatory power of the current models is limited, the results thus far strongly suggest that raags do consistently elicit specific emotions that are linked to musical properties. The responses did not differ significantly for enculturated and non-enculturated listeners, suggesting that musical rather than cultural factors are dominant.
\end{abstract}

\section{BACKGROUND}

Raag literally means "that which colors the mind". It is a melodic abstraction around which almost all North Indian classical music (NICM) is organized. A raag is most easily explained as a collection of melodic gestures and a technique for developing them. The gestures are sequences of notes that are often inflected with various micro-pitch alterations and articulated with an expressive sense of timing. Longer phrases are built by joining these melodic atoms together.

NICM uses approximately one hundred raags, of which fifty are common. Despite micro-tonal variation, the tones in any given raag conform to a subset of the twelve chromatic pitches of a standard just-intoned scale. There are theoretically thousands of scale types; in practice, however, raags conform to a much smaller set of scales, and many of the most common raags share the same set of notes.

By building phrases as described above, a tonal hierarchy is created. Some tones appear more often in the basic phrases, or are sustained longer. Indian music theory has a rich vocabulary for describing the function of notes in this framework. The most stressed note is called the vadi and the second most stressed, traditionally a fifth or fourth away, is called the samvadi. There are also less commonly used terms for tones on which phrases begin and end. A typical summary of a raag includes its scale type (that), vadi and samvadi. A pitch-class distribution (PCD), which gives the relative frequency of each scale degree, neatly summarizes this information.

The performance context of raag music is essentially monophonic, although vocalists will usually be shadowed by an accompanying melody instrument. The rhythmic accompaniment of the tabla is also present in metered sections. There is usually an accompanying drone that sounds the tonic and fifth using a harmonically rich timbre.

\section{RELATED WORK}

The emotional qualities of raags have been discussed extensively in NICM music theory. Traditionally, this music is said to evoke seven basic emotions: sadness, romance, peace, strength/courage, anger, dispassion, devotion (Bhatkande 1934). In theory, each raag elicits a unique emotional state (rasa) consisting of one or more of these basic emotions.

For Western music, a variety of studies have been undertaken since the late $19^{\text {th }}$ century to define what emotions music elicits, and to understand the musical factors that underly them. These studies have followed a basic experimental paradigm in which musical excerpts are presented to listeners who are then asked to respond by verbally describing their emotional states, rating emotions on a quantitative scale, or some other measurement. Musical stimuli are chosen so that certain characteristics can be systematically varied, such as timbre, tempo and tonality. Correlations between responses and musical structure are then observed, and are interpreted to account for some aspect of the listener reactions. 
A common pitfall in such experiments is the fundamental difficulty of isolating the factor that is being tested. Real musical excerpts rarely vary in just one parameter, while artificial stimuli that can be systematically varied often will not generalize, lacking the "ecological validity" of real music. Most importantly, the difficulty of quantifying a person's actual emotional state, either through observation or by eliciting verbal or other responses, makes such research extremely challenging. Despite this, the field has produced a body of work that suggests consistent relationships between musical structure and emotional response (Balkwill and Thompson 1999). In general, two broad categories of emotional responses have been found, relating to valence (happy-sad) and activity (vigorous-calm). For example, fast tempo has been shown to correlate consistently with positive valence and activity; the same has been shown for major (versus minor) tonality. A thorough overview of the field of emotion and music can be found in Juslin and Sloboda (2001).

Although the connection between raag and emotion is assumed to be systematic by nearly all enculturated listeners and performers, very little empirical work relating to emotion in NICM has been published. In one study, NICM musicians were asked to play short excerpts that conveyed either joy, sadness, anger, or peace. Listeners not familiar with NICM were asked to identify the predominant emotion. The recognition of intended emotions was mostly successful except for peace (Balkwill and Thompson 1999).

\section{MOTIVATION}

The current work seeks to characterize the emotional responses of listeners to different raags and the extent to which listeners report similar experiences; furthermore, if responses differ systematically by raag, it seeks to identify some of the musical factors that underly these responses. In NICM, all pitch-related activity is structured by the choice of raag. Each raag is traditionally associated with several basic emotions. For example, Raag Yaman is said to evoke peace, happiness, and devotion. Performers and listeners of NICM often report that the essence of raag-based music is the evocation of mood brought about by one or more related emotions, and that different raags reliably elicit different emotional states. In many cases, however, the more traditional descriptions are not accepted, or are thought of as rudimentary. Despite the central role of emotion in raag theory, almost no empirical work has been undertaken to systematically analyze listener response to different raags.

\section{SURVEY DESIGN}

For the purpose of collecting a large number of responses, we created an online survey in which participants were asked to respond to five raag excerpts in terms of "how listening to each raag made you feel." The survey can be found at http: / / www . paragchordia.com/survey

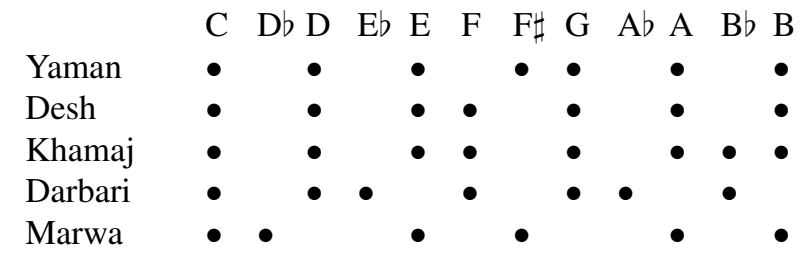

Table 1. Summary of scale degrees used by raags in database. Notes are listed with $\mathrm{C}$ as the tonic.

and the raag excerpts can be heard at http://www . paragchordia.com/research. Some basic demographic information was collected, specifically age, sex, level of musical training (if any), and familiarity with NICM (if any). For each excerpt, there was both a free response with the directions to "describe how it makes you feel in as much detail as possible," and a series of six value sliders (ranging from zero to one hundred) corresponding to pre-chosen emotional adjectives: happy, sad, tense, peaceful, romantic, and longing. The adjectives were chosen based on preliminary research by the author suggesting that these might best capture the dominant emotional axes. Further, the first four roughly represent the valence and activity judgments discussed above.

Participants were asked to "adjust the slider to indicate how well you feel that the word applies to the raag excerpt you just heard." The order in which each of these sliders appeared was randomized for each raag presented. Each participant was presented with the same set of five raags in a random order. The specific excerpt for each raag was chosen at random from three possibilities, one played on sarod and two on sitar, both traditional plucked string instruments. All excerpts were approximately one minute long, unaccompanied, and from alap, a slower, arrhythmic introductory section of a raag performance. Participants had the option of replaying each segment an unlimited number of times. Without knowing in advance how much data we would be able to collect, it was impossible to determine an appropriate dimensionality for the data we sought. Therefore, an attempt was made to strike a balance, leading to the decision to limit the number of slider-values to six and include a free-response section as well. This was also the reasoning that determined on the one hand the presentation of the same five raags to each participant, while generalizing slightly by the inclusion of three audio files for each raag.

\section{SURVEY RESULTS}

A total of 280 survey responses were collected. Participants were recruited from online NICM forums, as well as through the Georgia Institute of Technology music department. $29 \%$ of the respondents were female and $71 \%$ male, with a median age of 24 . The respondents described their familiarity with NICM as "none" (11\%), "a little"(24\%), "somewhat"(29\%), "very"(32\%) and "expert"(4\%). 

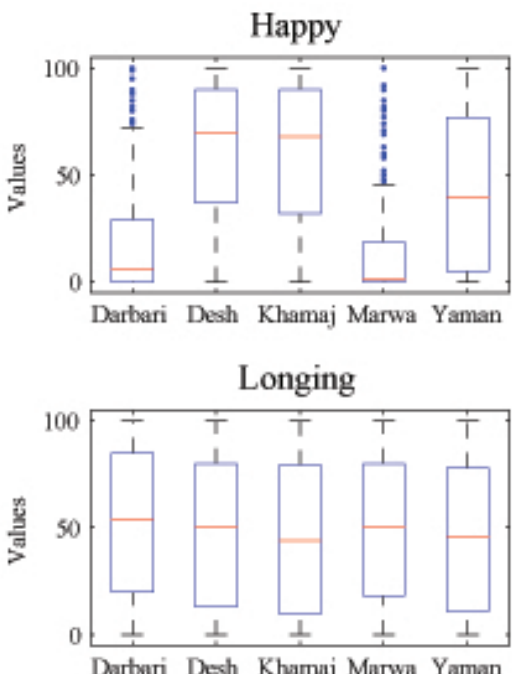

Peaceful

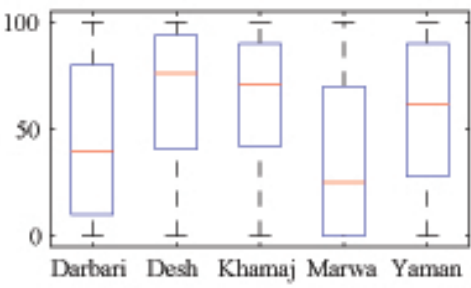

Tense

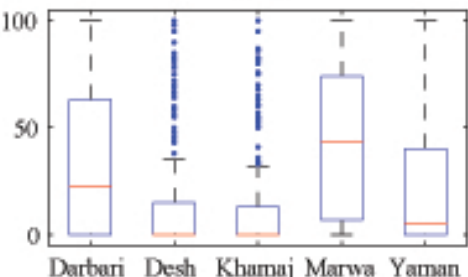

Sad

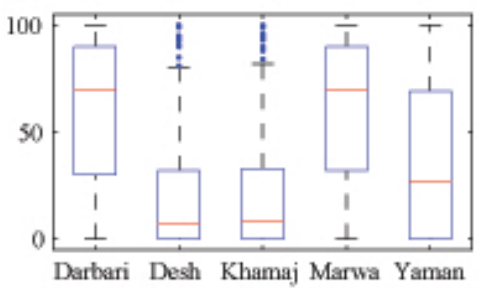

Romantic

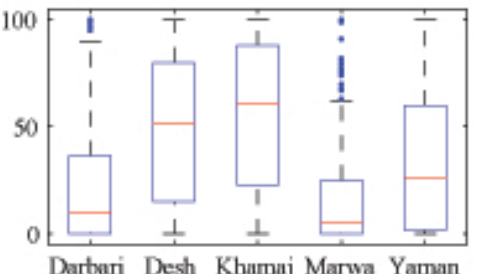

Figure 1. Summary of quantitative survey responses. Box and whisker plots for each emotion are shown. The $25^{\text {th }}$ to $75^{\text {th }}$ percentile is indicated by the box, the median by the horizontal line in the middle of the box. The range of the data is shown by the whiskers with outliers notated with hatch marks. It can be seen that Desh and Khamaj form one reponse cluster, and Marwa and Darbari another.

\subsection{Content Analysis of Free Responses}

The descriptive language of the free responses indicated the intensity and complexity of listeners' emotions. The style ranged from lists of words and phrases describing simple emotions to lengthy and even poetic passages. A sampling is shown in Table 4.

Due to the fact that many of the free responses consisted of fragments or lists of words, highly sophisticated content analysis was not particularly relevant; instead, we focus on lower-level analysis, primarily word histograms.

Initially, simple histograms of terms appearing in each free response section were tabulated. Limiting the results to relevant descriptive terms, the most common for each raag were collected and are shown in Table 2. Standard variations of each word (e.g. "happy" and "happiness") were treated as examples of the same root word.

Terms were also grouped according to various categories of meaning, in an attempt to both give a broader view of the responses and to capture information buried in the "long tail" of infrequently used but clearly related terms. For example, words such as "bliss", "exultation", "happy", "joyful", and "ecstasy" were grouped together under "Joy \& Happiness". Relevant semantic groupings were based on a modified version of a standard content analysis dictionary. As before, the most common occurrences were tabulated and the results are displayed in Table 3 .

The tables clearly show a common pattern of responses for certain raags. Raags Khamaj and Desh are associated with positively valenced concepts such as "joy \& happiness," whereas Darbari and Marwa were consistently associated with "melancholy \& sadness." Interestingly, $Y a$ - man was equally associated with "melancholy \& sadness" and "joy \& happiness." The results show that, although each raag has a strong valence, a variety of other emotions are also present, suggesting that listeners' responses are indeed complex.

\subsection{Quantitative Responses}

Figure 1 shows the distribution of quantitative (slider) responses for each emotion and raag. For happy, sad, tense, and romantic, three raag clusters are apparent. Desh and Khamaj are strongly positively valenced (happy, romantic and not tense) and Marwa and Darbari are negatively valenced (sad, not romantic, tense) with Yaman falling between these poles. Multiple comparison of means confirms that differences between these three clusters for these three emotions are significant $(p<.05)$ but that differences within clusters are not significant. The "longing" values show no statistically significant differences by raag, however all the raags clearly brought out this emotion. For the "peaceful"emotion there are again three clusters: Marwa is least peaceful followed by Darbari and then a cluster containing Yaman, Khamaj and Desh.

\section{FEATURE SELECTION}

In order to explore possible predictors for these emotional reactions, several features were extracted from the audio excerpts. Some of these features have previously been shown to be highly effective in raag classification (Chordia and Rae 2007), thus we sought to investigate their ability to explain emotional responses. We hypothesized that survey responses would in part be predicted by the pitches 


\begin{tabular}{|c|c|c|c|c|c|c|c|c|c|}
\hline \multicolumn{2}{|c|}{ Darbari } & \multicolumn{2}{|c|}{ Desh } & \multicolumn{2}{|c|}{ Khamaj } & \multicolumn{2}{|c|}{ Marwa } & \multicolumn{2}{|c|}{ Yaman } \\
\hline Freq. & Term & Freq. & Term & Freq. & Term & Freq. & Term & Freq. & Term \\
\hline 75 & sad & 59 & happy & 53 & happy & 74 & sad & 46 & sad \\
\hline 19 & peaceful & 41 & peaceful & 36 & peaceful & 16 & serious & 42 & peaceful \\
\hline 14 & deep & 19 & romantic & 31 & sad & 14 & good & 38 & happy \\
\hline 13 & calm & 17 & sad & 24 & romantic & 14 & peaceful & 13 & good \\
\hline 10 & longing & 15 & soothing & 13 & soothing & 14 & longing & 12 & calm \\
\hline 10 & serious & 11 & nice & 10 & good & 13 & deep & 11 & longing \\
\hline 10 & beautiful & 10 & pleasant & 9 & longing & 10 & tense & 8 & serious \\
\hline 9 & good & 10 & patriotic & 9 & love & 7 & emotional & 7 & romantic \\
\hline 8 & happy & 9 & playful & 9 & relax & 7 & happy & 7 & relax \\
\hline 5 & sombre & 9 & remind & 6 & calm & 6 & soothe & 6 & devotional \\
\hline
\end{tabular}

Table 2. Simple word histograms for each Raag

\begin{tabular}{|c|c|c|c|c|}
\hline Darbari & Desh & Khamaj & Marwa & Yaman \\
\hline Semantic Cat. & Semantic Cat. & Semantic Cat. & Semantic Cat. & Semantic Cat. \\
\hline 134 melancholy \& sadness & 115 joy \& happiness & 103 joy \& happiness & 133 melancholy \& sadness & 71 melancholy \& sadness \\
\hline 21 joy $\&$ happiness & 41 love \& affection & 54 melancholy \& sadness & 28 tension & 69 joy \& happiness \\
\hline 16 pains & 32 melancholy \& sadness & 37 love \& affection & 15 sympathy \& compassion & 16 emotions \\
\hline 15 emotion & 14 pleasures & 13 emotions & 12 pains & 16 love \& affection \\
\hline 12 beauty & 13 composure & 10 composure & 12 joy \& happiness & 12 beauty \\
\hline 11 tension & 12 beauty & 9 pleasures & 11 emotion & 9 composure \\
\hline 11 love \& affection & 11 hope \& optimism & 7 passions & 10 love \& affection & 8 tension \\
\hline 10 sympathy \& compassion & 6 emotion & 7 hope \& optimism & 7 anger \& indignation & 8 pleasures \\
\hline 8 despair \& resignation & 6 desires & 5 desires & 7 emotionalities & 6 passions \\
\hline
\end{tabular}

Table 3. Semantic category histograms for each Raag. Words with a similar meaning were assigned to a common semantic category.

Darbari: "Emptiness. I'm tumbling down a deep abyss. Weightless, then maybe. Dark and surrounding. Fall."

"It feels like pain, an agony that is long lasting and is happening at the time that the music is playing, a time of hardship."

Desh: "Absolutely fresh.... Clearing all the bad thoughts... Gives fresh meaning to life. Sounds serene. Very Peaceful i felt."

"Pure, unblemished...Something white as milk, offering to take you in, clean all your sins...Compassionate and loving, but in a distant sort of way."

Khamaj: "It so reminds me of the blossoming of flowers and prosperity... the happy chuckles of newborns... the pride of their mothers..."

"spring time. birds chirping. sun is shining. love. a mother is telling her child a story. the child is smiling. good times are upon us."

Marwa: "my reaction to this raaga was almost sexual. I felt desire. I felt the urge to look beautiful and dress up in heavy gold jewelery. felt very aware of my body. at times I felt a strange anger and the desire to control somebody else. i felt very powerful. i felt like a woman."

"very depressing...felt like crying...someone was going away... describes life in some way....the ups and downs."

Yaman: "I feel like a butterfly. The wind is streaking past me, and colors are awash in the air. Melodious colors." "A combination of moods. It looks relaxed most of the time and ruminating about something. It seems to turn a little angry occasionally as though an unhappy event was inadvertently recollected."

Table 4. A sample of free responses 
used and their relative weights, as represented by PCDs. In particular, it was expected that the relative prevalence of certain major and minor intervals would correspond to positively and negatively valenced responses.

Further, it was thought that sequential pairs of pitches, pitch class dyad distributions (PCDDs), would have a noticeable correlation. Extrapolating from Huron (Huron 2006), we hypothesized that the degree of flexibility in PCDDs would correspond to a sense of tension and longing. Flexibility was calculated as the entropy of the PCDD distributions, where a low entropy would correspond to an excerpt having highly determined, or leading, note transitions, possibly leading to a greater sense of longing.

A number of other features were considered as well, notably the overall dissonance of a given excerpt, calculated as the sensory dissonance. This feature was thought likely to correspond to negative emotions, although as calculated it might give excessively large values to spectrally rich sounds. The overall note density and spectral centroids for each excerpt were also determined.

\subsection{Pitch Detection and Pitch Class Distributions}

Pitch detection was done using a version of the Harmonic Product Spectrum (HPS) algorithm (Sun 2000). Each segment was divided into $40 \mathrm{~ms}$ frames, using a Gaussian window. The frames were overlapped by $75 \%$, so that the pitch was estimated every $10 \mathrm{~ms}$.

The PCDs were calculated by taking histograms of the pitch-tracks. The bins corresponded to each note of five octaves of a chromatic scale centered around the tonic for that segment. Specifically, the ratios of the just-intoned scale and the tonic frequency were used to calculate the center of each bin, and the edges were determined as the $\log$ mean. The five octaves were then folded into one and the values normalized to create a pitch-class distribution. This nulled any significance of octave errors in the HPS algorithm.

\subsection{Note Density}

The average note density for each excerpt was calculated from detected note onsets. Onsets were found by thresholding a complex detection function (DF) (Duxbury, Bello, Davies, and Sandler 2003). The excerpt was divided into 128 sample regions, overlapped $50 \%$ using a rectangular window, and the DFT of each region was computed and used to construct the DF. Adaptive thresholding, proportional to the median over a sliding window, was used to choose the peaks to be labeled as onsets.

\subsection{Pitch Class Dyad Distributions}

Pitch class dyad distributions were calculated for each excerpt. The detected onsets were used to segment the pitchtracks into notes. Each note was then assigned a pitchclass label: first the raw pitch estimates were discretized by assigning to each the center value of the bins defined for the pitch histogram, and then the mode was calculated for each note. The label of the corresponding chromatic pitch was assigned to that note. This process dealt quite effectively with variations due to micro-pitch structure, attacks, and errors by the detection algorithm. The octaves were folded into one as with the PCDs. The pitch-classes were then arranged in groups of two (bi-grams), or in musical terms, dyads. The entropy of the PCDD for each excerpt was then calculated.

\subsection{Sensory Dissonance}

The presence in a sound of partials which fall within the critical band for frequency resolution is the key indicator of dissonance, according to the tonotopic theory of sensory dissonance. A value for this was calculated by performing pairwise comparisons between all detected partials in each segment, and weighting the detected subcritical-band intervals by their relative amplitudes. This calculation was performed using an algorithm developed by Kameoka and Kuriyagawa (Kameoka and Kuriyagawa 1969).

\subsection{Timbral Features}

The center of mass in the frequency domain (spectral centroid) was calculated for each excerpt.

\section{ANALYSIS}

An initial demographic analysis showed that responses were not significantly different by age, sex, or familiarity with Indian music.

Multiple regression analysis was undertaken to determine how well the PCDs, subjective dissonance, and sensory dissonance features explained the emotion ratings. A linear model was built using these features for each of the quantitative emotional response types (with the exception of "longing", which was excluded because no statistically significant differences were found between raags). For example, the slider values for "happy" were modeled as

$$
\text { happy }_{i}=\beta_{1} x_{1}^{i}+\beta_{2} x_{2}^{i}+\beta_{3} x_{3}^{i}++\beta_{n} x_{n}^{i}
$$

where $x_{k}^{i}$ is the $\mathrm{k}^{\text {th }}$ feature and $\beta_{k}^{i}$ is the corresponding regression coefficient for the $\mathrm{i}^{\text {th }}$ observation. Stepwise regression was performed to determine the subset of features to be included in the final model.

Tables 5 and 6 show the beta values, that is the standardized regression coefficients, for the each of the features, as well as the $R^{2}$ statistic for the total model. All coefficients presented have $p$ values of less than .01. All variables in the model were normalized to have a mean of zero and a standard deviation of one, thus avoiding difficulties in comparing the scales of the independent variables. The $R^{2}$ statistic measures the total variance accounted for by the model and varies between 0 (random) and 1 (perfect fit). Of course, if measurement error is high, then the model will not be meaningful. The individual 

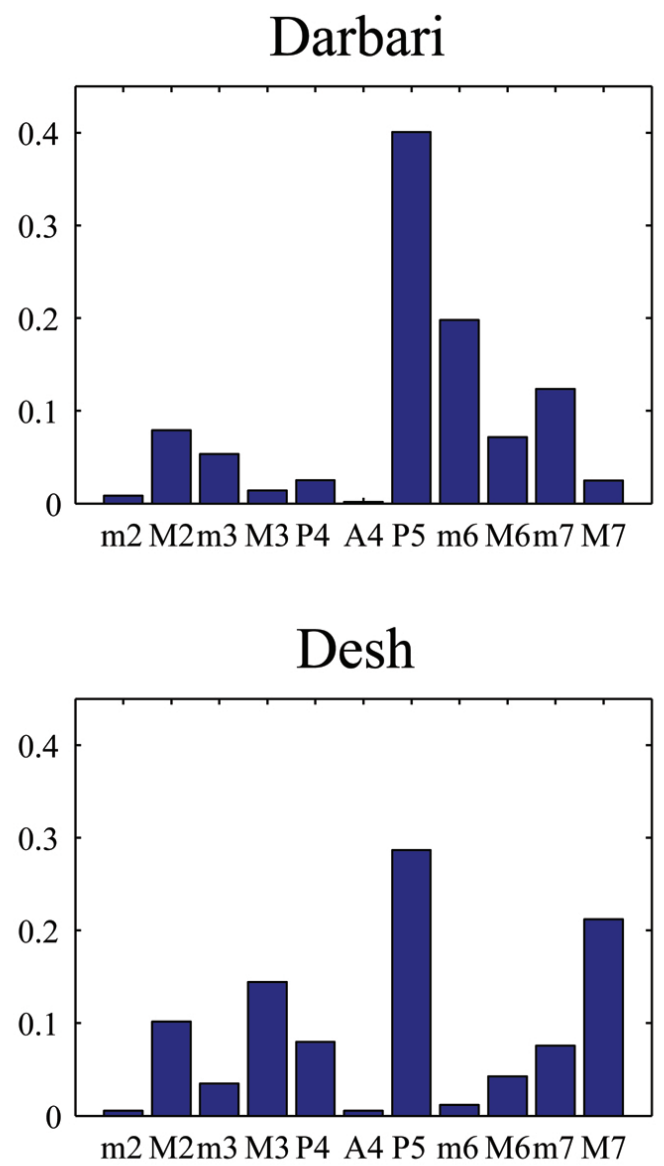

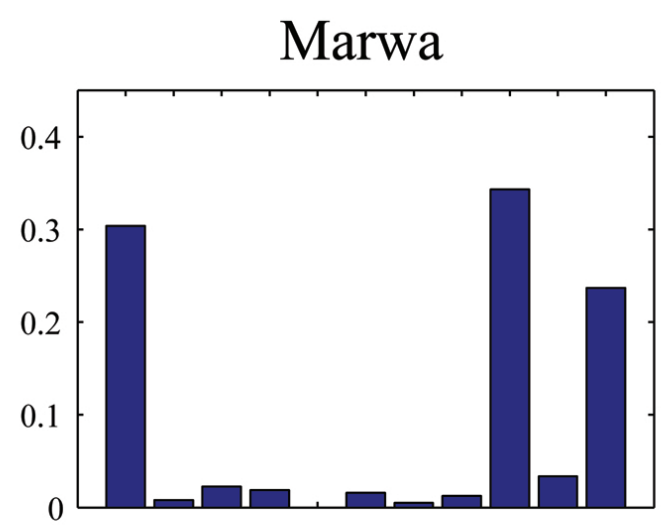

m2 M2m3 M3 P4 A4 P5 m6 M6m7 M7

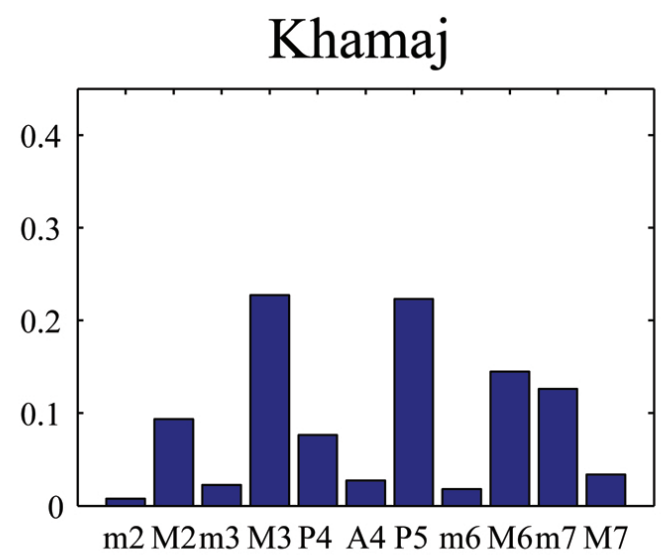

Figure 2. Pitch-class distributions for raags Desh and Khamaj

correlation between each feature and the emotion, which varies between -1 and 1 , is also shown.

Additionally the variance inflation factor (VIF) for each feature is shown, giving a measure of the multicollinearity of the independent variables. This occurs when one of the variables is well approximated by a linear combination of the other variables, as is likely to be the case for PCD values. The VIF is calculated by regressing each variable using the remaining independent variables, and is computed as $\frac{1}{1-R^{2}}$. If the $R^{2}$ value is high, it indicates that the variable is multicollinear. In typical applications, VIF values above a threshold of 10 indicate that a variable should be removed. Interpretation of models with multicollinear variables is difficult as the relative contribution of each cannot easily be read from the beta values and the reliability of the estimate decreases (the size of the confidence interval increases for that coefficient). In some cases, multicollinearity can reverse the expected sign of the regression coefficient. For example, in Table 5 in the "happy" model, the Major $3^{\text {rd }}$ coefficient is negative despite a strong positive correlation; it can be seen, however, that the variable is highly multicollinear, with a VIF of 7.74. Because scale tones in raags may be highly correlated, it is important to be aware of this potential confound. Stepwise regression partially avoids this problem by automatically selecting features that give the greatest incremental contribution to explaining the variance of the dependent variable. In this way, only one of a set of highly correlated features is typically included. Nevertheless, highly correlated variables may remain in the model, making VIF useful for interpretation.

Table 5 shows that "happy" and "sad" are modeled best, with $R^{2}$ values of .34 and .28 respectively, whereas the model is only weakly predictive in the case of "peaceful" (.11) and "tense" (.16). Unsurprisingly, "happy" responses are negative correlated with the minor $2^{\text {nd }}(\beta=$ $-.13)$, minor $3^{\text {rd }}(\beta=-.16)$, minor $6^{\text {th }}(\beta=-.43)$, and minor $7^{\text {th }}(\beta=-.37)$. Surprisingly, the Major $3^{\text {rd }}$ is also negatively correlated with a $\beta$ value of -.13 . As mentioned before, however, the Major $3^{\text {rd }}$ has a high VIF value; examination of the correlation coefficients shows that it is strongly correlated with the Perfect $4^{\text {th }}(.84)$, which is positively related to "happy" responses. "Sad" responses show an opposite pattern, positively related to the minor $2^{\text {nd }}(\beta=$ $.14)$, minor $3^{\text {rd }}(\beta=.35)$, and minor $6^{\text {th }}(\beta=.20)$, and negatively to the Perfect $4^{\text {th }}(\beta=-.20)$. Many of the other models loosely conform to the idea that rags with "minor" intervals are negatively valenced (sad, tense) and raags with "major" intervals are positively valenced (happy, peaceful). 


\begin{tabular}{|c|c|c|c|}
\hline Нарру" & Мо & $\mathbf{T}$ & 3 \\
\hline feature & $\beta$ & corr & VIF \\
\hline $\operatorname{minor} 2^{\text {nd }}$ & -0.13 & -0.29 & 4.23 \\
\hline $\operatorname{minor} 3^{\text {rd }}$ & -0.16 & -0.09 & .86 \\
\hline Major $3^{\text {rd }}$ & -0.13 & 0.38 & 7.74 \\
\hline Perfect $4^{\text {th }}$ & 0.13 & 0.37 & 5.66 \\
\hline $\operatorname{minor} 6^{\text {th }}$ & -0.43 & -0.25 & 6.0 \\
\hline Major $6^{\text {th }}$ & -0.35 & -0.28 & 2.3 \\
\hline Major $7^{\text {th }}$ & -0.37 & -0.03 & 8.1 \\
\hline nsory dissonance & 0.13 & 0.06 & 2.7 \\
\hline PCDD entropy & 0.18 & 0.38 & 2.6 \\
\hline
\end{tabular}

\begin{tabular}{|c|c|c|c|}
\hline \multicolumn{4}{|c|}{ "Sad" Regression Model - Total $R^{2}=.28$} \\
\hline feature & $\beta$ & corr & VIF \\
\hline $\operatorname{minor} 2^{\text {nd }}$ & 0.14 & 0.26 & 2.11 \\
\hline Major $2^{\text {nd }}$ & -0.16 & -0.14 & 1. \\
\hline minor $3^{\text {rd }}$ & 0.35 & 0.09 & 2.1 \\
\hline Perfect $4^{\text {th }}$ & -0.20 & -0.29 & 1. \\
\hline $\operatorname{minor} 6^{\text {th }}$ & 0.20 & 0.28 & 1.1 \\
\hline Major $6^{\text {th }}$ & 0.20 & 0.25 & 2. \\
\hline spectral centroid & -0.18 & -0.10 & 1. \\
\hline note density & -0.15 & -0.11 & 1. \\
\hline
\end{tabular}

Table 5. Summary of regression models for "happy" and "sad". For each feature we report the standardized regression coefficients, the bivariate correlation, and the variance inflation factor measure of multicollinearity.

$\begin{array}{crrr}\text { "Peaceful" Regression Model }- \text { Total } R^{2}=.11 \\ \text { scale deg } & \text { beta } & \text { corr } & \text { VIF } \\ \text { Major } 2^{\text {nd }} & 0.22 & 0.11 & 3.32 \\ \text { minor } 3^{\text {nd }} & -0.24 & -0.05 & 2.76 \\ \text { Major } 3^{\text {rd }} & 0.22 & 0.22 & 4.69 \\ \text { Perfect 4 } & 0.15 & 0.23 & 4.63 \\ \text { Perfect } 5^{\text {th }} & 0.15 & 0.10 & 1.20 \\ \text { Major } 7^{\text {th }} & 0.23 & 0.03 & 1.88 \\ \text { PCDD entropy } & -0.16 & 0.19 & 3.46\end{array}$

\begin{tabular}{|c|c|c|c|}
\hline \multicolumn{4}{|c|}{ "Tense" Regression Model - Total $R^{2}=.16$} \\
\hline scale deg & beta & corr & VIF \\
\hline $\operatorname{minor} 2^{\text {nd }}$ & 0.10 & 0.25 & 1.92 \\
\hline Major $2^{\text {nd }}$ & -0.21 & -0.15 & 1.71 \\
\hline $\operatorname{minor} 3^{\text {rd }}$ & 0.24 & 0.08 & 1.55 \\
\hline Major $3^{\text {rd }}$ & -0.14 & -0.26 & 1.45 \\
\hline Perfect $5^{\text {th }}$ & -0.20 & -0.18 & 1.74 \\
\hline $\operatorname{minor} 6^{\text {th }}$ & 0.14 & 0.12 & 1.45 \\
\hline
\end{tabular}

Table 6. Summary of regression models for "peaceful" and "tense"

The entropy of the PCDD distribution was a significant factor in the "happy" and "peaceful" models, positively related in the former and negatively related in the latter. We had hypothesized that low PCDD entropy values would correspond to tension and longing and higher values to a greater sense of flexibility. The effect shown here is weakly consistent with this prediction.

It is important to note that the relationship between PCDD entropy and the emotional characteristics we are testing is likely non-linear. Values outside the range represented in this study might well be expected to elicit different reactions. One might expect very low values, corresponding to predictability and repetition, to elicit "peaceful" and "sad" reactions. Very high values, on the other hand, might correspond to unpredictability and hence elicit feelings of "tension" and "stress". However, in the middle of the range, where most of the musical excerpts used here lie, low entropy corresponds to raags with a relatively fixed phraseology, creating a sense of tendency rather than repetition, and high entropy corresponds to raags with a greater degree of flexibility, conveying more variability than instability. If correlations observed in the above models are valid, this is the most likely explanation.

Two additional models were developed to see if a more parsimonious explanation of the data could be given. The first used the total strength of the minor $2^{\text {nd }}$, minor $3^{\text {rd }}$, and minor $6^{\text {th }}$ in the raag as the independent variable, a measure of the total degree of "minorness". The adjusted $R^{2}$

$$
R_{a}^{2}=1-\left(1-R^{2}\right) \frac{n-1}{n-p-1}
$$

$\begin{array}{rrrr} & \text { Full } & \text { PCD only } & \text { Minor } \\ \text { Happy } & 0.34 & 0.27 & 0.07 \\ \text { Sad } & 0.28 & 0.22 & 0.06 \\ \text { Peaceful } & 0.10 & 0.10 & 0.02 \\ \text { Tense } & 0.16 & 0.13 & 0.05 \\ \text { Romantic } & 0.21 & 0.18 & 0.08\end{array}$

Table 7. Comparison of three regression models. The total adjusted $R^{2}$ value for each model is shown.

which allows comparison between models with a different number of independent variables, is reported in Table 7. The second model considered only the PCD features. The full model was significantly more explanatory. Although the full model suggested that a feature that combined the minor $2^{\text {nd }}$, minor $3^{\text {rd }}$ and minor $6^{\text {th }}$ in total measure of "minorness" might capture most of the information in the PCD, this was not the case. The PCD features explained an additional $10-20 \%$ of variation as compared with the single "minor" feature.

\section{DISCUSSION}

Survey responses have shown that different raags evoke a clearly differentiated set of emotional reactions. Free responses tended to cluster strongly in particular adjectival categories based on raag, and quantitative responses were significantly different by raag for all emotions except "longing". Thus, a substantial step has been taken towards empirical verification of the nature and reliabil- 
ity of emotional responses to raag. Importantly, responses did not vary systematically by familiarity with NICM suggesting that listeners were not simply referring to culturally determined concepts, but responding to underlying features of the music.

The analysis, although preliminary, suggests that responses are in part attributable to pitch-class statistics; the prevalence of certain scale degrees is useful in predicting the valence of the emotional responses. The data suggest that the entropy of the PCDD and the spectral centroid are also important. These are undoubtedly just a few of the many factors that influence listener responses. As more data are collected it will be possible to more fully examine other factors.

It is important to note that these models are currently merely suggestive. In none of the cases were they highly predictive, with a maximum of $34 \%$ of the variance accounted for. Because the goal here was explanatory rather than classificatory, the models were not verified on an independent data set. As with any task that forces respondents to verbalize primarily non-verbal mental states, there is significant measurement error due to the inherent unnaturalness of the task and an imperfect ability to map the verbal space. It is also possible that much of the true emotional feel of the music is lost in the projection onto simple emotions such as "happy" and "sad". Although it is likely that some aspect of raags can be effectively captured by mapping onto these axes, it is also likely that it is a gross simplification of the actual emotional experience.

\section{CONCLUSIONS AND FUTURE WORK}

We have reported the results of the first empirical survey of listeners' emotional reactions to raag music. We have established that responses exhibit clear patterns and have identified several musical features which partially explain them. Future work will consider a larger set of raags and provide listeners with more dimensions along which to evaluate their emotional experiences. As more data is collected, it will be possible to test a greater number of musical features and more robustly assess their validity.

\section{References}

Balkwill, L. L. and W. F. Thompson (1999). A crosscultural investigation of the perception of emotion in music: Psychophysical and cultural cues. Music Perception 17, 43-64.

Bhatkande, V. (1934). Hindusthani Sangeet Paddhati. Sangeet Karyalaya.

Chordia, P. and A. Rae (2007). Raag recognition using pitch-class and pitch-class dyad distributions. In Proceedings of International Conference on $\mathrm{Mu}$ sic Information Retrieval.

de la Cuadra, P., A. Master, and C. Sapp (2001). Efficient pitch detection techniques for interactive mu- sic. In Proceedings of the International Computer Music Conference, pp. 403-406.

Duxbury, C., J. P. Bello, M. Davies, and M. Sandler (2003). A combined phase and amplitude based approach to onset detection for audio segmentation. In Proc. of the 4th European Workshop on Image Analysis for Multimedia Interactive Services (WIAMIS-03), London, pp. 275-280.

Huron, D. (2006). Sweet Anticipation: Music and the Psychology of Expectation. MIT Press.

Juslin, P. N. and J. A. Sloboda (2001). Music and Emotion: Theory and Research. Oxford University Press.

Kameoka, A. and M. Kuriyagawa (1969). Consonance theory, part i: Consonance of dyads. Journal of the Acoustical Society of America 45(6), 1451-1459.

Sun, X. (2000). A pitch determination algorithm based on subharmonic-to-harmonic ratio. In In Proc. of International Conference of Speech and Language Processing. 